\title{
Expressing a Robot's Confidence with Motion-based Artificial Subtle Expressions
}

Seiji Yamada

National Institute of Informatics / SOKENDAI

2-1-2 Hitotsubashi, Chiyoda,

Tokyo, 101-8430 Japan

seiji@nii.ac.jp
Takanori Komatsu

Meiji University

1-1 Kandasurugadai, Chiyoda

Tokyo, 101-8301 Japan

tkomat@acm.org

\section{Kotaro Funakoshi}

Honda Research Institute

Japan Co., Ltd.

8-1 Honcho, Wako

Saitama, 351-0188 Japan

funakoshi@jp.honda-ri.com

\section{Mikio Nakano}

Honda Research Institute

Japan Co., Ltd.

8-1 Honcho, Wako

Saitama, 351-0188 Japan

nakano@jp.honda-ri.com

kby@shinshu-u.ac.jp

Copyright is held by the author/owner(s)

CHI 2013 Extended Abstracts, April 27-May 2, 2013, Paris,

France.

ACM 978-1-4503-1952-2/13/04.

\begin{abstract}
In this paper, motion-based Artificial Subtle Expression (ASE) as a novel implementation of ASE is described, in which a robot expresses confidence in its advice to a human. Confidence in advice is one of robot's useful internal states, and it is an important goal to develop a practical and inexpensive methodology to correctly express it. To achieve this goal, we propose motion-based ASE in which a robot slowly hesitates by turning to a human before giving advice with low confidence. We conducted experiments to evaluate the effectiveness of motion-based ASE with participants, and obtained promising results.
\end{abstract}

\section{Author Keywords}

Expression of a robot; ASE; Human-Agent Interaction $(\mathrm{HAl}) ; \mathrm{HRI}$

\section{ACM Classification Keywords}

H.5.2 [[Information Interfaces And Presentation]]: User Interfaces - Theory and methods;

\section{General Terms}

Experimentation; Design; Human Factors.

\section{Introduction}

In Human-Agent Interaction (HAI) and Human-Robot

Interaction (HRI), it is important that a robot informs a 
human of its internal state, including its confidence in processed results, emotion, and so on [7]. In this work, we focus on a robot's confidence in the information that it informs to a human, and we develop a simple and effective method for expressing this confidence with less cognitive load. We consider confidence to be one of the robot's most significant internal states that can be expressed along with various pieces of information that a robot informs a human of, e.g., a robot should express confidence in its advice during human decision making.

In human communication, a speaker's confidence in the content of what they are saying is frequently expressed as non-verbal information, such by facial expressions, pitch, and gestures, to complement the main verbal communication [6]. In particular, subtle expression [1] of such non-verbal information is known to enrich human communication, and the engineering implementation methodology of such subtle expression for virtual agents, robots, and appliances is called "Artificial Subtle Expression" (ASE) [8]

In this paper, we apply ASE to a robot so that it is able to express its confidence in advice given to a human during decision making. We developed a turning-around behavior and a motion-based ASE (motion-ASE for short) that can be implemented simply by changing the velocity of the behavior. Then, we conducted experiments with a treasure chest guessing task with participants to evaluate the effectiveness of the motion-ASE.

Various studies on expressing a robot's internal state have been done thus far for HRI. With a mobile robot, facial expressions are indicated on the top of the robot [11]. Most of these studies were an investigation of the relationship between LED color and the expressed emotions [9]. Almost all of the studies dealt with the emotions of a robot, not its confidence.

Subtle expressions and non-verbal communication have also been implemented in robots. There are studies on the effective head movements of humanoid robots [4] and experiments to change the movement velocity of a shopping cart robot [3] for human-robot communication. One of these researchers worked on expressing confidence with robots. A lot of studies needed a humanoid robot and such a robot is very expensive and hard to control precisely.

ASEs have been applied to various domains $[8,5]$ thus far. We believe this work significantly extends ASE's applicability to new domains, robot motions excluding beep sounds [8], and LED lighting [5].

Motion-ASE: Expressing Confidence with Motion

Robot's Confidence and Hesitation Behavior Since the processing in a robot contains various noise from the real environment, the information expressed from the robot to a human frequently has uncertainty. This uncertainty is significantly caused by a limitation in the robot's ability, not by the ambiguity of human interpretation [10]

Thus, a robot needs to inform a human of its confidence in HRI situations. Since confidence significantly influences human decision making, a robot's expression of confidence is important. We propose motion-ASE for mobile robots so that they can express confidence with simple representations and low cognitive load required for human interpretation. 
In human communication, information with low confidence is frequently expressed with slow and pausing motions as hesitation [2]. Thus, we implemented motion-ASE by using hesitation behavior and having a robot slowly turn around to face a human as is done in HRI where a robot turns to a human before advising him/her. We consider this behavior to be executed in various HRI situations. We assume that, since slowly turning around can be used to express a robot's hesitation to a human, the human feels the confidence in the advice is lower than when the robot turns fast. Although there are various ways to use ASE to express hesitation, we consider this slow turn to be one of the most suitable expressions because it is simple, does not require any additional equipment, and the cost of implementation is very low. Furthermore motion-ASE has the possibility to be extended to various robots for the same reason. The concrete implementation of the hesitation behavior and experimental evaluation will be discussed in the next section.

\section{ASE Design Policy}

We applied ASE design policy [8] to implement the ability of a robot to express its confidence in its advice to a human during decision making because ASE is suitable for a mobile robot to inform a human of its binary internal state (i.e., high/low confidence in this work) in an inexpensive way [8]. ASE has the following four requirements as the design policy, and they should be satisfied to realize ASE. The first two are design requirements that should be satisfied in the design phase, and the remaining two are function requirements that should be experimentally evaluated after the implementation of ASE [8]. We will discuss how motion-ASE satisfies the above requirements in the later sections.
(A1) Simple: ASE is implemented in a single modality.

(A2) Complementary: ASE does not interfere with communication's main protocol (e.g. verbal communication).

(A3) Intuitive: ASE is intuitively understandable.

(A4) Accurate: ASE conveys the designer's intended meanings accurately.

Since motion-ASE has the single visual modality of a robot turning to a human, (A1) is satisfied, and (A2) is also satisfied because turning to a human is executed quietly. The remaining (A3) and (A4) will be evaluated later in the discussion.

\section{Experiments}

We conducted experiments with participants to evaluate the effectiveness of motion-ASE. In this work, we set a mobile robot to slowly turn around to face a human as the motion-ASE described before. We used the speed of the turning as a parameter for expressing the robot's confidence in its advice. A human sometimes turns quickly to another to intentionally express strong agreement and sometimes turns slowly to express hesitation. Thus, we believe the speed of the turning can be a parameter to be investigated for motion-ASE. Thus we introduced two conditions: fast-motion and slow-motion.

As another parameter to be investigated for expressing confidence, we introduced delay time, which is the delay before the robot starts to give advice. Since the robot's advice is given to a human just after turning around, the delay time of the fast-motion condition is shorter than that of the slow-motion condition. To investigate how only the delay time might influence the expression of the robot's confidence without turning around, we introduce short-wait and long-wait conditions without the turning 


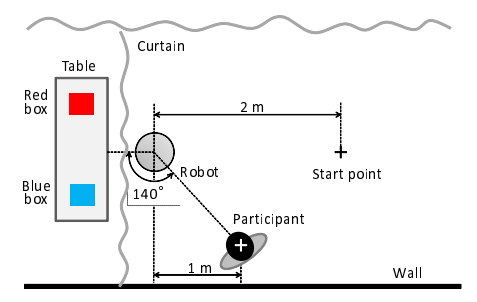

Figure 1: Experimental environment.

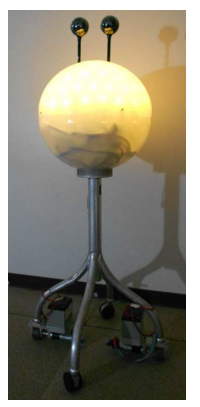

Figure 2: Robot used in the experiments (yellow lighting).

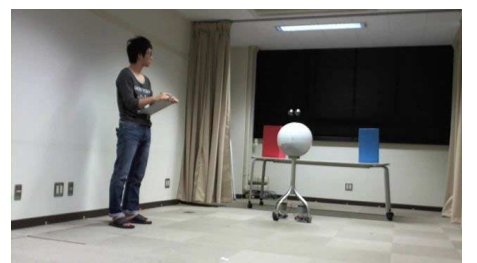

Figure 3: Snapshot of Experiment at the Robot's behavior (R2). around. To clarify the requirements for the most effective expression, we used four conditions: fast-motion, slow-motion, short-wait, and long-wait for the experiments.

\section{Experimental Task, Environment and Robot}

We used a treasure chest guessing task as a representative experimental task of human decision making in which participants were required to repeatedly guess which of two boxes (i.e., a red or blue box) on a table contained a treasure. In each trial, a robot gave advice to the participants by saying the color (i.e., red or blue) of the box that it guessed contained the treasure by using speech synthesis. The participants did not know how the robot could guess the location of the treasure.

We used between-participant design. A participant repeatedly tried to guess the location of the treasure under one of the four experimental conditions so that he/she could not compare the behaviors of the different conditions. Participants were not told about the correct location of the treasure after each guess so that they could not estimate the probability that the robot's advice was correct by using the feedback history.

Figure 1 shows the experimental environment. The experimental room was surrounded by curtains. The curtain in front of the boxes was closed at the beginning of every trial, and then an experimenter opened the curtain. Figure 2 shows the robot used through all the experiments. It had two wheels, and its two motors were controlled by a laptop PC through a USB connection. Dead reckoning and computer vision were used to correct the localization of the robot and the turning-around behavior. The robot autonomously behaved without WOZ.
Procedure, Evaluation Indexes, and Participants Participants were told that a treasure was set in either the red or blue box at the beginning of the trial and asked to choose which box they expected to contain the treasure after hearing the robot's advice because the robot and they were cooperative partners. No knowledge of the robot's behaviors except that of the robot's advice was informed to the participants. Also, they were required to score their estimated confidence of the robot's advice by using a seven-point-scale value after every trial. Each participant performed this trial ten times under one of the four conditions. The participant's and the robot's behaviors, $(\mathrm{P})$ and $(\mathrm{R} n)$ are described as follows.

First, an experimenter hid the two boxes from each participant by closing the curtain and put a treasure into one of them. Then, he/she would say "Next problem" to the participant.

(R1) The robot went to the front of the table. The moving speed was $25 \mathrm{~cm} / \mathrm{sec}$.

(R2) It stopped there for two seconds to think. (Figure 3 shows a snapshot.)

(R3) It blinked yellow for one second after finishing thinking.

(R4) It expressed confidence in its advice. In the fast or slow-motion condition, the robot quickly or slowly turned $140^{\circ}$ to the participant for two or eight seconds, respectively. In the short or long-wait condition, it only stopped in front of the table for two or eight seconds, respectively.

(R5) It said "Red" or "Blue" as advice to the participant.

(P) The participant answered "Red" or "Blue" to indicate the expected location of the treasure after hearing the robot's advice, and then he/she scored 


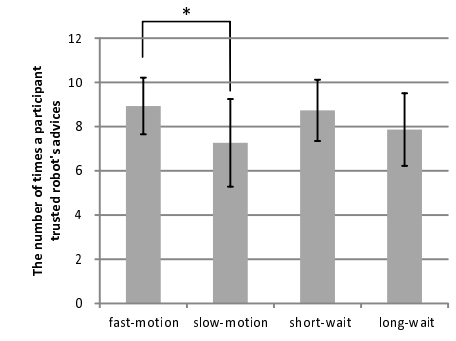

Figure 4: The number of times a participant accepted robot's advices.

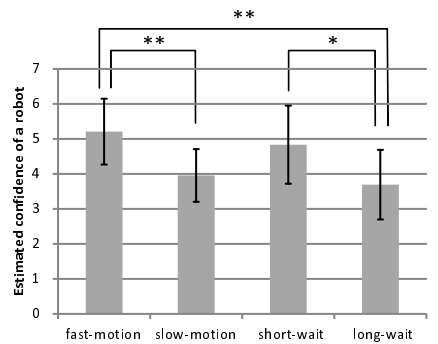

Figure 5: Estimated confidence on the robot's advice. the estimated confidence by using a seven-point-scale value.

(R6) The robot returned to the initial position.

We measured the number of times a participant accepted the robot's advice for behavioral evaluation. We also measured the estimated confidence of the robot's advice evaluated by the participants by using a seven-point-scale value as a subjective and more detailed evaluation. We expected "excellent expression" to outperform the others in both evaluations

For this experiment, we used 60 Japanese undergraduate students in the computer engineering department of Gifu University in Japan. They were 18 to 24 years old, and equally divided into four groups with almost the same average age. In regard to the gender balance of the participants, there were 44 males and 16 females. This balance was similar to that in the department, and there was no intended control for the gender balance.

\section{Results}

Figure 4 shows the number of times the robot's advice was accepted by the participants (averages and SD). The mean score of the fast-motion condition was 8.93 (SD = 1.28 ), that of the slow-motion condition was 7.27 (SD $=$ 1.98 ), that of the short-wait condition was 8.73 (SD = 1.39 ), and that of the long-wait condition was 7.86 (SD = 1.64). Figure 5 shows participants' ratings of estimated confidence for each condition (averages and SD). The mean score of the fast-motion condition was 5.21 (SD = .94 ), that of the slow-motion condition was 3.95 (SD = $.75)$, that of the short-wait condition was 4.83 (SD = 1.12 ), and that of the long-wait condition was 3.69 (SD = $1.00)$
We performed ANOVAs to find the difference between the conditions in terms of the number of times a participant accepted the robot's advice and in terms of the estimated confidence in the robot's advice. Significant differences were found in the number of acceptances $[F(3,56)=$ $3.54, p<.05]$ with the effect size $\eta^{2}=.16$ and the estimated confidence $[F(3,56)=8.33, p<.01]$ with $\eta^{2}=$ .31. Furthermore, multiple comparisons made by using Tukey's method found a significant difference between the fast-motion and slow-motion conditions $(p<.05)$ in terms of the number of acceptances, between the fast-motion and slow-motion conditions $(p<.01)$, between the fast-motion and long-wait conditions $(p<.01)$, and between the short-wait and long-wait conditions $(p<.05)$ in the estimated confidence.

\section{Discussion}

In this paper, we introduced the behavior of a robot slowly turning around to face a human as motion-ASE. As seen from the number of times participants accepted the robot's advice (Figure 4) and from the estimated confidence (Figure 5), significant differences between the fast-motion and slow-motion conditions were found in both of the two evaluations. Thus, by using the fast-motion and slow-motion that the mobile robot used to express its confidence to a human, the participants could correctly interpret high/low-confidence from fast/slow-motion expressions and could act based on this interpretation. As a result, we experimentally confirmed the effectiveness of motion-ASE.

Meanwhile, as seen from the results of the long/short-wait conditions, although significant difference was not found in the number of times a participant accepted the robot's advice (Figure 4), it was found in the estimated confidence (Figure 5). In contrast to fast/slow-motion 
expressions having a difference both in the behavioral evaluation and the subjective evaluation, long/short-wait expressions had it only in the subjective evaluation. Thus, we conclude that, although the long/short-wait expressions might be applicable to expressing a robot's confidence, fast/slow-motion as a motion-ASE is more suitable for such expressions.

As described in the ASE Design Policy, while ASE requirements $(A 1)$ and $(A 2)$ were satisfied in the design phases of motion-ASE, (A3) and (A4) needed to be evaluated after the experiments. In the experiments, although participants had no knowledge of expressions and the between-participant design made the evaluation absolute, they could interpret the motion-ASE . Thus, we believe that ASE requirement (A3) was satisfied. Also, since their interpretation was found to be significantly correct from the experimental results, requirement (A4) was satisfied too

\section{Conclusion}

In HAI and HRI, a robot's confidence in its advice is a useful internal state that should be correctly expressed to a human, and it is an important goal to develop a practical methodology to express it. For this goal, we proposed motion-ASE as a novel implementation of ASE in which a robot hesitates to slowly turn-around to face a human before giving advice with low confidence. We conducted experiments to evaluate the effectiveness with participants, and obtained results that support the effectiveness.

\section{References}

[1] C. Bartneck and J. Reichenbach. Subtle emotional expressions of synthetic characters. Int. Journal of Human-Computer Studies, 62(2):179-192, 2005.
[2] E. S. Reed, et al. On the nature and significance of microslips in everyday activities. Ecological Psychology Studies, 4(1):51-66, 2009.

[3] H. Hüttenrauch, et al. Evaluation of robot body movements supporting communication. In Proc. of 2nd International Symposium on New Frontiers in Human-Robot Interaction, pages 42-49, 2010.

[4] C. T. Ishi, C. Liu, H. Ishiguro, and N. Hagita. Head motions during dialogue speech and nod timing control in humanoid robots. In HRI'10, pages 293-300, 2010

[5] K. Funakoshi, et al. Non-humanlike spoken dialogue: a design perspective. In SIGDIAL'10, pages 176-184, 2010

[6] A. Kendon. Do gestures communicate? a review. Research on Language \& Social Interaction, 27(3):175-200, 1994

[7] M. Scheutz, P. Schermerhorn, and J. Kramer. The utility of affect expression in natural language interactions in joint human-robot tasks. In HRI'06, pages 226-233, 2006.

[8] T. Komatsu, et al. Artificial subtle expressions: Intuitive notification methodology of artifacts atlanta. In CHI'10, pages 1941-1944, 2010.

[9] K. Terada, A. Yamauchi, and A. Ito. Artificial emotion expression for a robot by dynamic color change. In RO-MAN'12, pages 314-321, 2012.

[10] S. Thrun. Probabilistic robotics. Communications of the ACM, 45(3):52-57, 2002.

[11] J. E. Young, M. Xin, and E. Sharlin. Robot expressionism through cartooning. In HRI'07, pages 309-316, 2007. 